\title{
Nonorthogonality and $\kappa$-Dependence Eccentricity of Polarized Electromagnetic Waves in CPT-Even Lorentz Violation
}

\author{
Thiago Prudêncio ${ }^{1}$ and Humberto Belich ${ }^{2}$ \\ ${ }^{1}$ Coordination of Science \& Technology (CCCT/BICT), Federal University of Maranhão (UFMA), 65080-805 São Luís, MA, Brazil \\ ${ }^{2}$ Departamento de Física e Química (DFQ), Federal University of Espirito Santo (UFES), 29060-900 Vitoria, ES, Brazil \\ Correspondence should be addressed to Thiago Prudêncio; thprudencio@gmail.com
}

Received 14 December 2016; Accepted 6 March 2017; Published 20 March 2017

Academic Editor: Shi-Hai Dong

Copyright (c) 2017 Thiago Prudêncio and Humberto Belich. This is an open access article distributed under the Creative Commons Attribution License, which permits unrestricted use, distribution, and reproduction in any medium, provided the original work is properly cited. The publication of this article was funded by SCOAP ${ }^{3}$.

\begin{abstract}
We discuss the modified Maxwell action of a $K_{F}$-type Lorentz symmetry breaking theory and present a solution of Maxwell equations derived in the cases of linear and elliptically polarized electromagnetic waves in the vacuum of CPT-even Lorentz violation. We show in this case that the Lorentz violation has the effect of changing the amplitude of one component of the magnetic field, while leaving the electric field unchanged, leading to nonorthogonal propagation of electromagnetic fields and dependence of the eccentricity on $\kappa$-term. Further, we exhibit numerically the consequences of this effect in the cases of linear and elliptical polarization, in particular, the regimes of nonorthogonality of the electromagnetic wave fields and the eccentricity of the elliptical polarization of the magnetic field with dependence on the $\kappa$-term.
\end{abstract}

\section{Introduction}

The recent discovery of the Higgs boson at the LHC established a research program that aims to explain the physics of fundamental interactions, like excitations manifested from fundamental fields, ending a program of tremendous experimental predictions, the Standard Model (SM) of particle physics. This model has the merit of unifying the weak force and electromagnetism by the Anderson-Higgs mechanism. Nevertheless, it is known that the description of the SM without neutrino mass is unsatisfactory. Recent experiments indicate that the electrons have a bound for the electric dipole moment [1-3] and the SM predicts that the electron is a punctual particle. By these experimental results we understand the necessity of investigating physics beyond the Standard Model. Kostelecký and Samuel [4] showed that interactions in the context of the string field theory could lead to spontaneous breaking of the Lorentz symmetry. Such a scenario cannot exist in the regime of SM, but can be the key for explaining regimes where quantum gravity and the early stages of the universe bring reminiscent breaking effects in the limits of validity of SM. In these other regimes, structure of spacetime and the corresponding particle physics are affected in their symmetries. A spontaneous breaking of Lorentz symmetry can then be extended beyond the known scenarios of QED and QCD [5-9].

Lorentz violation theories are in the framework of Standard Model Extension (SME) [10, 11]. From the SM perspective, Lorentz symmetry breaking induces an asymmetry in the structure of spacetime, implying for instance that vacuum Maxwell equations has to be changed by nontrivial contributions. One important feature of LVT in the domain of electrodynamics is the possibility of violating Lorentz symmetry without breaking gauge symmetry. One of the first models in this sense was carried out by the Carroll-FieldJackiw model [12] where breaking of the Lorentz invariance is realized by the inclusion of an additional term in the Maxwell Lagrangian, while preserving the gauge symmetry. General case of $\kappa$-electrodynamics was introduced in [13], determining the energy momentum tensor and basic properties of the plane wave solutions of $\kappa$-electrodynamics. A more indepth study of the model and its plane wave solutions was performed in [14], where equations of motion in Maxwell form are derived. A special case of the $\kappa$-parameter was 
discussed in [15] that also constructs the plane wave solutions including with polarization vectors. The idea that the special case of the $\kappa$-parameter acts like an effective metric has been used widely in the literature [16].

The presence of terms that violate the Lorentz symmetry imposes at least one privileged direction in the spacetime [1727]. Nowadays, studies in relativistic quantum effects [2834] that stem from a nonminimal coupling with Lorentz symmetry breaking [35-41] have opened the possibility of investigating new implications in quantum mechanics that this violator background can promote [42-44].

In this work, we discuss free electromagnetic fields with a particular type of Lorentz symmetry violation: the vacuum reduction of a $K_{F}$-type Lorentz symmetry breaking theory [45] in the absence of complex scalar field $\varphi$ and $\lambda$-parameter associated with a $\lambda\left|\phi^{4}\right|$ are zero. The modified dispersion laws of electromagnetic waves are obtained in [16] with two fourvectors as violating background; that is, they are interpreted as preferred directions in spacetime. We investigate the scenario of the modified Maxwell equations resulting from this CPT-even scenario of Lorentz violation. In this case, we consider the modified Maxwell equations in the vacuum of a $K_{F}$-type Lorentz symmetry breaking where the Lorentz violation is manifested only by the presence of a $\kappa^{\mu \nu \rho \sigma}$ tensor. We then consider a solution of Maxwell equations derived in the cases of linear and elliptical polarization electromagnetic waves in the vacuum of CPT-even Lorentz violation scenario and investigate the effect of the amplitude changing of the amplitudes electromagnetic wave fields and regimes of nonorthogonality of the electromagnetic wave fields and the eccentricity of the elliptical polarization with dependence on the $\kappa$-term.

The structure of this paper is as follows: in Section 2 we discuss the modified Maxwell action by the presence of the tensor $\kappa_{a b c d}$; in Section 3, the Lagrangian; in Section 4, the effective metric; in Section 5, the momentum energy tensor for the modified Maxwell action; and, in the Section 6, the Maxwell equations from the modified Maxwell Lagrangian. In Section 7, we present our conclusions.

\section{The Tensor $\kappa_{a b c d}$}

We started off our analysis with the Maxwell action in the presence of the Lorentz violating term

$$
\Sigma=\int d^{4} x\left\{-\sqrt{-g} \frac{1}{4} F_{\mu \nu} F^{\mu \nu}\right\}+\Sigma_{k}
$$

where the part responsible for the Lorentz violation is given by

$$
\Sigma_{k}=-\frac{1}{4} \int d^{4} x \sqrt{-g}\left(\kappa^{\mu \nu \rho \sigma} F_{\mu \nu} F_{\rho \sigma}\right)
$$

The tensor $\kappa_{\mu \nu \sigma \rho}$ is CPT-even; that is, it does not violate the CPT-symmetry. Although the violation of the CPTsymmetry implies that the Lorentz invariance is violated [46], the reverse is not necessarily true. The action with the presence of $\kappa_{a b c d}$ breaks the Lorentz symmetry in the sense that the tensor $\kappa_{a b c d}$ has a nonnull vacuum expectation value. Besides, the tensor $\kappa_{a b c d}$ has the same properties of the Riemann tensor, as well as an additional double-traceless condition. This tensor possesses the following symmetries:

$$
\begin{aligned}
\kappa_{a b c d} & =\kappa_{[a b][c d]}, \\
\kappa_{a b c d} & =\kappa_{c d a b}, \\
\kappa_{a b}^{a b} & =0 .
\end{aligned}
$$

By following [47-49], we can write the tensor $\kappa_{a b c d}$ in terms of a traceless and symmetric matrix $\widetilde{\kappa}_{a b}$ as

$$
\kappa_{a b c d}=\frac{1}{2}\left(\eta_{a c} \widetilde{\kappa}_{b d}-\eta_{a d} \widetilde{\kappa}_{b c}+\eta_{b d} \widetilde{\kappa}_{a c}-\eta_{b c} \widetilde{\kappa}_{a d}\right),
$$

and $\eta^{a c}$ is the metric tensor with signature $(+;-,-,-)$.

By defining a normalized parameter four-vector $\xi^{a}$, which satisfies the conditions: $\xi_{a} \xi^{a}=1$ for the timelike case and $\xi_{a} \xi^{a}=-1$ for the spacelike case; thus, we can decompose the tensor $\tilde{\kappa}_{a b}$ as

$$
\widetilde{\kappa}_{a b}=\kappa\left(\xi_{a} \xi_{b}-\frac{\eta_{a b} \xi^{c} \xi_{c}}{4}\right)
$$

where

$$
\kappa=\frac{4}{3} \widetilde{\kappa}^{a b} \xi_{a} \xi_{b} .
$$

We deal with a Lorentz symmetry violating tensor $k_{\mu \nu \kappa \lambda}$ in such a way that the CPT-symmetry is preserved. It has been shown in [45] that a particular decomposition [47, 49] of the tensor $\kappa_{\mu \nu \rho \sigma}$ produces a modification on the equations of motion of the electromagnetic waves due to the presence of vacuum anisotropies, which gives rise to the modified Maxwell equations. As a consequence, the anisotropy can be a source of the electric field and, then, Gauss's law is modified. Besides, the Ampere-Maxwell law is modified by the presence of anisotropies and it has a particular interest in the analysis of vortices solutions since it generates the dependence of the vortex core size on the intensity of the anisotropy.

\section{Modified Maxwell Lagrangian}

We start with the following modified Maxwell Lagrangian from the CPT-even $K_{F}$-type Lorentz symmetry breaking theory

$$
\begin{aligned}
& \mathscr{L}_{\operatorname{modMax}} \\
& \quad=-\sqrt{-g}\left(\frac{1}{4} F_{\mu \nu} F_{\rho \sigma} g^{\mu \rho} g^{\nu \sigma}+\frac{1}{4} \kappa^{\mu \nu \rho \sigma} F_{\mu \nu} F_{\rho \sigma}\right) .
\end{aligned}
$$

Using the description of $\kappa^{\mu \nu \rho \sigma}$ in tetrad fields

$$
\kappa^{\mu \nu \rho \sigma}=\kappa^{a b c d} e^{\mu}{ }_{a} e^{\nu}{ }_{b} e^{\rho} e_{c}^{\sigma}{ }_{d}
$$

and the decomposition in the nonbirefringent sector eq. (4), we can write the modified contribution as

$$
\begin{aligned}
\kappa^{\mu \nu \rho \sigma} F_{\mu \nu} F_{\rho \sigma}= & \frac{1}{2}\left(\eta^{a c} \widetilde{\kappa}^{b d}-\eta^{a d} \widetilde{\kappa}^{b c}+\eta^{b d} \widetilde{\kappa}^{a c}-\eta^{b c} \widetilde{\kappa}^{a d}\right) \\
& \cdot e^{\mu}{ }_{a} e^{\nu} e^{\rho} e_{c} e^{\sigma}{ }_{d} F_{\mu \nu} F_{\rho \sigma} .
\end{aligned}
$$


Advances in High Energy Physics

3

Taking into account the relation between metric and tetrad fields

$$
g^{\mu \rho} e^{\nu}{ }_{b} e_{d}^{\sigma}=\eta^{a c} e^{\mu}{ }_{a} e^{\nu} e^{\rho}{ }_{c} e^{\sigma}{ }_{d}
$$

we also have

$$
\begin{gathered}
\kappa^{\mu \nu \rho \sigma} F_{\mu \nu} F_{\rho \sigma}=\frac{1}{2}\left(g^{\mu \rho} \widetilde{\kappa}^{b d} e^{\nu}{ }_{b} e^{\sigma}{ }_{d}-g^{\mu \sigma} \widetilde{\kappa}^{b c} e^{\nu}{ }_{b} e^{\rho}\right. \\
\left.+g^{\nu \sigma} \widetilde{\kappa}^{a c} e^{\mu}{ }_{a} e^{\rho}{ }_{c}-g^{\nu \rho} \widetilde{\kappa}^{a d} e^{\mu}{ }_{a} e^{\sigma}{ }_{d}\right) F_{\mu \nu} F_{\rho \sigma} .
\end{gathered}
$$

Considering the tensor

$$
\widetilde{\kappa}^{a b}=\kappa\left(\xi^{a} \xi^{b}-\frac{\eta^{a b} \xi^{c} \xi_{c}}{4}\right),
$$

we can rewrite

$$
\begin{aligned}
& \kappa^{\mu \nu \rho \sigma} F_{\mu \nu} F_{\rho \sigma}=\frac{1}{2} \\
& \cdot \kappa\left[g^{\mu \rho}\left(\xi^{b} \xi^{d} e^{\nu}{ }_{b} e^{\sigma}{ }_{d}-\frac{\eta^{b d} e_{b}^{\nu} e^{\sigma}{ }_{d} \xi^{e} \xi_{e}}{4}\right)\right. \\
& -g^{\mu \sigma}\left(\xi^{b} \xi^{c} e^{\nu}{ }_{b} e^{\rho}{ }_{c}-\frac{\eta^{b c} e^{\nu} e^{\rho}{ }_{c} \xi^{\mu} \xi_{\mu}}{4}\right) \\
& +g^{\nu \sigma}\left(\xi^{a} \xi^{c} e^{\mu}{ }_{a} e^{\rho}{ }_{c}-\frac{\eta^{a c} e^{\mu}{ }_{a} e^{\rho}{ }_{c} \xi^{e} \xi_{e}}{4}\right) \\
& \left.\quad-g^{\nu \rho}\left(\xi^{a} \xi^{d} e^{\mu}{ }_{a} e^{\sigma}{ }_{d}-\frac{\eta^{a d} e^{\mu}{ }_{a} e^{\sigma}{ }_{d} \xi^{e} \xi_{e}}{4}\right)\right] F_{\mu \nu} F_{\rho \sigma} .
\end{aligned}
$$

We also have the following relation for tetrad fields

$$
g^{\nu \rho}=\eta^{b c} e_{b}^{v} e_{c}^{\rho}=e^{\nu b} e_{c}^{\rho}
$$

which will lead to the following contribution:

$$
\begin{aligned}
& \kappa^{\mu \nu \rho \sigma} F_{\mu \nu} F_{\rho \sigma}=\frac{1}{2} \kappa\left[g^{\mu \rho}\left(\xi^{\nu} \xi^{\sigma}-\frac{g^{\nu \sigma} \xi^{e} \xi_{e}}{4}\right)\right. \\
& -g^{\mu \sigma}\left(\xi^{\nu} \xi^{\rho}-\frac{g^{\nu \rho} \xi^{e} \xi_{e}}{4}\right)+g^{\nu \sigma}\left(\xi^{\mu} \xi^{\rho}-\frac{g^{\mu \rho} \xi^{e} \xi_{e}}{4}\right) \\
& \left.-g^{\nu \rho}\left(\xi^{\mu} \xi^{\sigma}-\frac{g^{\mu \sigma} \xi^{e} \xi_{e}}{4}\right)\right] F_{\mu \nu} F_{\rho \sigma} .
\end{aligned}
$$

We can also rewrite

$$
\begin{gathered}
\kappa^{\mu \nu \rho \sigma} F_{\mu \nu} F_{\rho \sigma}=\frac{1}{2} \kappa\left[\left\{g^{\mu \rho}\left(\xi^{\nu} \xi^{\sigma}-\frac{g^{\nu \sigma} \xi^{e} \xi_{e}}{4}\right)\right.\right. \\
\left.+g^{\nu \sigma}\left(\xi^{\mu} \xi^{\rho}-\frac{g^{\mu \rho} \xi^{e} \xi_{e}}{4}\right)\right\} F_{\mu \nu} F_{\rho \sigma} \\
-\left\{g^{\mu \sigma}\left(\xi^{\nu} \xi^{\rho}-\frac{g^{\nu \rho} \xi^{e} \xi_{e}}{4}\right)\right. \\
\left.\left.+g^{\nu \rho}\left(\xi^{\mu} \xi^{\sigma}-\frac{g^{\mu \sigma} \xi^{e} \xi_{e}}{4}\right)\right\} F_{\mu \nu} F_{\rho \sigma}\right] .
\end{gathered}
$$

Identifying the even symmetry in the terms $F_{\mu \nu} F_{\rho \sigma}$ in the pairs $\mu \sigma, \nu \rho$, and using the antisymmetry in the second term under the exchange $\rho \rightarrow \sigma, \sigma \rightarrow \rho$, we achieve

$$
\kappa^{\mu \nu \rho \sigma} F_{\mu \nu} F_{\rho \sigma}=2 \kappa\left[g^{\mu \rho}\left(\xi^{\nu} \xi^{\sigma}-\frac{g^{\nu \sigma} \xi^{e} \xi_{e}}{4}\right) F_{\mu \nu} F_{\rho \sigma}\right] .
$$

The effective Lagrangian can now be written as

$$
\begin{aligned}
& \mathscr{L}_{\text {modMax }}=-\sqrt{-g}\left(\frac{1}{4}\right. \\
& \left.\cdot F_{\mu \nu} F_{\rho \sigma}\left[g^{\mu \rho} g^{\nu \sigma}+2 \kappa g^{\mu \rho}\left(\xi^{\nu} \xi^{\sigma}-\frac{g^{\nu \sigma} \xi^{e} \xi_{e}}{4}\right)\right]\right) .
\end{aligned}
$$

4. Effective Metric

We can simplify the terms in the Lagrangian as follows:

$$
\begin{aligned}
& F_{\mu \nu} F_{\rho \sigma}\left[g^{\mu \rho} g^{\nu \sigma}+2 \kappa g^{\mu \rho}\left(\xi^{\nu} \xi^{\sigma}-\frac{g^{\nu \sigma} \xi^{e} \xi_{e}}{4}\right)\right] \\
& \quad=F_{\mu \nu} F_{\rho \sigma}\left[g^{\mu \rho} g^{\nu \sigma}+\left(2 \kappa g^{\mu \rho} \xi^{\nu} \xi^{\sigma}-\frac{\kappa g^{\mu \rho} g^{\nu \sigma} \xi^{e} \xi_{e}}{2}\right)\right]
\end{aligned}
$$

and rewrite

$$
\begin{gathered}
F_{\mu \nu} F_{\rho \sigma}\left(g^{\mu \rho} g^{\nu \sigma}+2 \kappa g^{\mu \rho}\left(\xi^{\nu} \xi^{\sigma}-\frac{g^{\nu \sigma} \xi^{e} \xi_{e}}{4}\right)\right) \\
=F_{\mu \nu} F_{\rho \sigma}\left(g^{\mu \rho}\left(g^{\nu \sigma}+\kappa \xi^{\nu} \xi^{\sigma}\right)\right. \\
\left.+\kappa \xi^{\mu} \xi^{\rho}\left[g^{\nu \sigma}-\frac{g^{\nu \sigma} g^{\mu \rho} \xi^{e} \xi_{e} / 2}{\xi^{\mu} \xi^{\rho}}\right]\right) .
\end{gathered}
$$

(14) The Lagrangian can then be written as follows:

$$
\begin{aligned}
& \mathscr{L}_{\operatorname{modMax}}=-\sqrt{-g} \frac{1}{4} F_{\mu \nu} F_{\rho \sigma}\left[g^{\mu \rho}\left(g^{\nu \sigma}+\kappa \xi^{\nu} \xi^{\sigma}\right)\right. \\
& \left.+\kappa \xi^{\mu} \xi^{\rho}\left(g^{\nu \sigma}-\frac{g^{\nu \sigma} g^{\mu \rho} \xi^{e} \xi_{e} / 2}{\xi^{\mu} \xi^{\rho}}\right)\right] .
\end{aligned}
$$

We can define the effective metric

$$
\tilde{g}_{\mu \nu}=g_{\mu \nu}-\frac{\kappa}{1+\kappa \xi \rho \xi_{\rho} / 2} \xi_{\mu} \xi_{\nu}
$$

and its inverse as

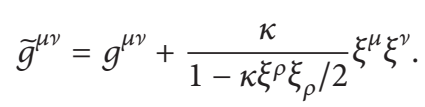

In order to prove the identity relation, we have to consider

$$
\begin{aligned}
\tilde{g}^{\mu \nu} \tilde{g}_{\mu \nu}= & \left(g^{\mu \nu}+\frac{\kappa}{1-\kappa \xi^{\rho} \xi_{\rho} / 2} \xi^{\mu} \xi^{\nu}\right) \\
& \cdot\left(g_{\mu \nu}-\frac{\kappa}{1+\kappa \xi \rho \xi_{\rho} / 2} \xi_{\mu} \xi_{\nu}\right) ;
\end{aligned}
$$


we can simplify

$$
\tilde{g}^{\mu \nu} \tilde{g}_{\mu \nu}=g^{\mu \nu} g_{\mu \nu}
$$

The Lagrangian can be written after some algebra as follows:

$$
\begin{gathered}
\mathscr{L}_{\text {modMax }}=-\sqrt{-g} \frac{1}{4} F_{\mu \nu} F_{\rho \sigma}\left\{\left(1-\frac{\kappa \xi^{\mu} \xi_{\mu}}{2}\right) \tilde{g}^{\mu \rho} \tilde{g}^{\nu \sigma}\right. \\
\left.-\frac{\kappa^{2}}{1-\kappa \xi^{\mu} \xi_{\mu} / 2} \xi^{\nu} \xi^{\sigma} \xi^{\mu} \xi^{\rho}\right\} .
\end{gathered}
$$

Taking the small $\kappa$ in the previous result, we can neglect the higher order terms and the Lagrangian will reduce to

$$
\mathscr{L}_{\text {modMax }}=-\sqrt{-g} \frac{1}{4} F_{\mu \nu} F_{\rho \sigma}\left[\left(1-\frac{\kappa \xi^{\rho} \xi_{\rho}}{2}\right) \widetilde{g}^{\nu \sigma} \widetilde{g}^{\mu \rho}\right] .
$$

We could also achieve the approximation for small $\kappa$ by means of the relation

$$
\begin{aligned}
& F_{\mu \nu} F_{\rho \sigma}\left(g^{\mu \rho}\left(g^{\nu \sigma}+\kappa \xi^{\nu} \xi^{\sigma}\right)\right. \\
& \left.+\kappa \xi^{\mu} \xi^{\rho}\left(g^{\nu \sigma}-\frac{g^{\nu \sigma} g^{\mu \rho} \xi^{e} \xi_{e} / 2}{\xi^{\mu} \xi^{\rho}}\right)\right) \\
& \quad F_{\mu \nu} F_{\rho \sigma}\left(g^{\mu \rho}\left(g^{\nu \sigma}+\kappa \xi^{\nu} \xi^{\sigma}\right)\right. \\
& \left.+\kappa \xi^{\mu} \xi^{\rho}\left(g^{\nu \sigma}+\kappa \xi^{\nu} \xi^{\sigma}\right)\right), \\
& \tilde{g}^{\mu \rho}=\left(g^{\mu \rho}+\kappa \xi^{\mu} \xi^{\rho}\right) \sim\left(g^{\mu \rho}+\frac{2 \kappa}{2+\kappa} \xi^{\mu} \xi^{\rho}\right)
\end{aligned}
$$

and then achieve the result

$$
\mathscr{L}_{\text {modMax }}=-\sqrt{-g}\left(1-\frac{1}{2} \kappa \xi^{\rho} \xi_{\rho}\right) \frac{1}{4} F_{\mu \nu} F_{\rho \sigma} \widetilde{g}^{\mu \rho} \widetilde{\mathfrak{g}}^{\nu \sigma} .
$$

\section{Momentum Energy Tensor}

The momentum energy tensor from the modified action can be written as

$$
\begin{aligned}
T_{\alpha \beta}= & g_{\alpha \beta}\left\{\frac{1}{4}\left(1-\frac{\kappa}{2} \xi^{2}\right) F_{\mu \nu} F^{\mu \nu}+\frac{\kappa}{2} \xi^{\nu} \xi^{\sigma} F^{\rho}{ }_{\nu} F_{\rho \sigma}\right\} \\
& +F^{\nu}{ }_{\beta} F_{\nu \alpha}\left(1+\frac{\kappa}{2} \xi^{2}\right)+\kappa \xi^{\nu} \xi^{\sigma} F_{\alpha \nu} F_{\beta \sigma} .
\end{aligned}
$$

The energy associated is then given by the following component:

$$
\begin{aligned}
T_{00}= & g_{00}\left\{\frac{1}{4}\left(1-\frac{\kappa}{2} \xi^{2}\right) F_{\mu \nu} F^{\mu \nu}+\frac{\kappa}{2} \xi^{\nu} \xi^{\sigma} F^{\rho}{ }_{\nu} F_{\rho \sigma}\right\} \\
& +F^{\nu}{ }_{0} F_{\nu 0}\left(1+\frac{\kappa}{2} \xi^{2}\right)+\kappa \xi^{\nu} \xi^{\sigma} F_{0 \nu} F_{0 \sigma} \\
= & 1\left\{\frac{1}{4}\left(1-\frac{\kappa}{2} \xi^{2}\right)\left(\mathbf{E}^{2}-\mathbf{B}^{2}\right)+\frac{\kappa}{2} \xi^{\nu} \xi^{\sigma} F^{\rho}{ }_{\nu} F_{\rho \sigma}\right\} \\
& +F^{\nu}{ }_{0} F_{\nu 0}\left(1+\frac{\kappa}{2} \xi^{2}\right)+\kappa \xi^{\nu} \xi^{\sigma} F_{0 \nu} F_{0 \sigma} .
\end{aligned}
$$

\section{Maxwell Equations from the Modified Maxwell Lagrangian}

The modified Maxwell equations in the vacuum of a $K_{F}$-type Lorentz symmetry breaking theory in the presence of the complex scalar field $\varphi$ and the $\lambda$-parameter associated with a $\lambda\left|\phi^{4}\right|$-theory obtained in [45] are

$$
\begin{aligned}
- & \left(1-\frac{\kappa \xi^{2}}{2}\right)\left(\partial_{\mu} F^{\mu \nu}\right) \\
= & \kappa \xi^{\nu} \xi^{\sigma} \partial^{\rho} F_{\rho \sigma}-\kappa g^{\nu \rho} \xi^{\mu} \xi^{\sigma} \partial_{\mu} F_{\rho \sigma} \\
& +i e\left(\varphi \partial^{\nu} \varphi^{*}-\varphi^{*} \partial^{\nu} \varphi\right)+2 e^{2} A^{\nu} \varphi^{*} \varphi .
\end{aligned}
$$

We write explicitly the modified Maxwell equations as follows:

$$
\begin{aligned}
- & {\left[\left(1-\frac{\kappa \xi^{2}}{2}-\kappa\left(\xi^{0}\right)^{2}\right) \nabla \cdot-\kappa \lambda \vec{\xi} \cdot\right] \mathbf{E} } \\
= & \kappa \xi^{0}\left(\partial^{0} \vec{\xi} \cdot \mathbf{E}+\vec{\xi} \cdot \nabla \times \mathbf{B}\right)+i e\left(\varphi \partial^{0} \varphi^{*}-\varphi^{*} \partial^{0} \varphi\right) \\
& +2 e^{2} \varphi^{*} \varphi \mathbf{\Phi}, \\
\nabla \times E & =-\frac{\partial \mathbf{B}}{\partial t} \\
\nabla \cdot \mathbf{B} & =0, \\
- & \left(1-\frac{\kappa \xi^{2}}{2}\right)\left(-\partial_{0} \mathbf{E}+\nabla \times \mathbf{B}\right) \\
= & \kappa \xi^{0}(\vec{\xi} \nabla \cdot-\kappa \lambda) \mathbf{E}-\kappa(\vec{\xi}+\kappa \lambda)(\vec{\xi} \cdot \nabla \times \mathbf{B}) \\
& -i e\left(\varphi \nabla \varphi^{*}-\varphi^{*} \nabla \varphi\right)+2 e^{2} \mathbf{A} \varphi^{*} \varphi .
\end{aligned}
$$

These are reduced to the same set of equations in the absence of the complex scalar field as we start from the modified Maxwell Lagrangian in the bilinear gauge action we derived

$$
\begin{aligned}
\Sigma_{g} & =\int d^{4} x\left\{-\frac{1}{4} F_{\mu \nu} F^{\mu \nu}\right. \\
& \left.-\frac{1}{4} 2 \kappa\left[\left(g^{\mu \rho}\left(\xi^{\nu} \xi^{\sigma}-\frac{g^{\nu \sigma} \xi^{e} \xi_{e}}{4}\right)\right) F_{\mu \nu} F_{\rho \sigma}\right]\right\} .
\end{aligned}
$$

In this case, we can understand the anisotropy generated by the kind of Lorentz violation we are considering. We obtain the generalized equation

$$
\begin{aligned}
& -\partial_{\mu}\left[\left(1-\frac{\kappa \xi^{e} \xi_{e}}{2}\right) F^{\mu \nu}\right] \\
& =\partial_{\mu}\left(\kappa F^{\mu \sigma} \xi_{\sigma} \xi^{\nu}\right)-\partial_{\alpha}\left(\kappa F^{\nu \sigma} \xi_{\sigma} \xi^{\alpha}\right) .
\end{aligned}
$$

In terms of the components $(\nu=0)$

$$
\begin{aligned}
-\nabla & {\left[\left(1-\frac{\kappa \xi^{e} \xi_{e}}{2}\right) \mathbf{E}\right] } \\
= & \partial_{0}\left(\kappa \xi^{0} \mathbf{E} \cdot \vec{\xi}\right)+\nabla \cdot\left(\kappa\left(\mathbf{E} \xi_{0}+\mathbf{B} \times \vec{\xi}\right) \xi^{0}\right) \\
& -\partial_{0}\left(\kappa \mathbf{E} \cdot \vec{\xi} \xi^{0}\right)-\nabla \cdot(\kappa \vec{\xi}(\mathbf{E} \cdot \vec{\xi}))
\end{aligned}
$$


and $(\nu=i)$ we have

$$
\begin{aligned}
\partial_{0}\left[\left(1-\frac{\kappa \xi^{e} \xi_{e}}{2}\right) \mathbf{E}\right]-\nabla \times\left[\left(1-\frac{\kappa \xi^{e} \xi_{e}}{2}\right) \mathbf{B}\right] \\
=\partial_{0}(\kappa \vec{\xi}(\mathbf{E} \cdot \vec{\xi}))+\partial_{j}\left(\kappa\left(E^{j}\right) \xi_{0} \vec{\xi}\right)+\nabla \\
\quad \cdot(\kappa(\vec{\xi} \times \mathbf{B}) \vec{\xi})-\partial_{0}\left(\kappa(\mathbf{E}) \xi_{0} \xi^{0}+\kappa(\vec{\xi} \times \mathbf{B}) \xi^{0}\right) \\
\quad-\partial_{j}\left(\kappa(\mathbf{E}) \xi_{0} \xi^{j}+\kappa(\vec{\xi} \times \mathbf{B}) \xi^{j}\right) .
\end{aligned}
$$

These equations take into account the possible dynamical variation of the background represented by the presence of $\xi^{e}$ and $\kappa$. In the static background we achieve the usual set of modified Maxwell equations in the vacuum of a $K_{F}$-type Lorentz symmetry breaking theory [45], in the absence of the complex scalar field $\varphi$, and the $\lambda$-parameter associated with a $\lambda\left|\phi^{4}\right|$-theory is zero and is reduced to modified Maxwell equations by the presence of the $K^{\mu \nu \rho \sigma}$ tensor. In this case, these equations are written

$$
\begin{aligned}
\nabla \cdot \mathbf{E}= & \frac{-\kappa \xi^{0}}{\left[1-\kappa \xi^{2} / 2-\kappa\left(\xi^{0}\right)^{2}\right]} \vec{\xi} \cdot \frac{\partial \mathbf{E}}{\partial t} \\
& +\frac{-\kappa \xi^{0}}{\left[1-\kappa \xi^{2} / 2-\kappa\left(\xi^{0}\right)^{2}\right]} \vec{\xi} \cdot \nabla \times \mathbf{B}, \\
\nabla \cdot \mathbf{B}= & 0, \\
\nabla \times \mathbf{E}= & -\frac{\partial \mathbf{B}}{\partial t}, \\
\nabla \times \mathbf{B}= & \frac{\partial \mathbf{E}}{\partial t}+\frac{\kappa \xi^{0} \vec{\xi} \nabla \cdot \mathbf{E}-\kappa \vec{\xi}(\vec{\xi} \cdot \nabla \times \mathbf{B})}{-\left(1-\kappa \xi^{2} / 2\right)} .
\end{aligned}
$$

Notice that these modified Maxwell equations are written in the vacuum by the presence of Lorentz violation in the electromagnetic field dynamics. A feature of this modification is that (39) and (40) are not changed.

The other two of the Maxwell equations, (38) and (41), are modified by the anisotropy generated by the kind of Lorentz violation term in which we are considering. The modified Gauss law (38) indicates that, to a nonstationary electrical field, we gain a charged anisotropic background. In the stationary case we have the usual vacuum Gauss law. In the case of the Ampere-Maxwell modified equation (41) we note that beyond the contribution of the electric field variation in the intensity of the magnetic field, we have a anisotropic contribution. In [45] we have a practical discussion on the influence of this background in (41) to the vortices solutions.

Considering a $\xi_{\mu}$ point in the following specific direction:

$$
\begin{aligned}
\xi^{0} & =0, \\
\vec{\xi} & =\widehat{\mathbf{x}}
\end{aligned}
$$

we have $\kappa=4 \widetilde{\kappa}^{11} / 3$ and the modified Maxwell equations are

$$
\begin{aligned}
\nabla \cdot \mathbf{E} & =0, \\
\nabla \cdot \mathbf{B} & =0, \\
\nabla \times \mathbf{E} & =-\frac{\partial \mathbf{B}}{\partial t}, \\
\nabla \times \mathbf{B} & =\frac{\partial \mathbf{E}}{\partial t}+\frac{\kappa(\nabla \times \mathbf{B})_{x}}{(1-\kappa / 2)} \widehat{\mathbf{x}} .
\end{aligned}
$$

In this configuration the only change is in the $x$ component of (46)

$$
(\nabla \times \mathbf{B})_{x}=\frac{\partial E_{x}}{\partial t}+\frac{\kappa(\nabla \times \mathbf{B})_{x}}{(1-\kappa / 2)}
$$

or

$$
(\nabla \times \mathbf{B})_{x}=\frac{\partial B_{z}}{\partial y}-\frac{\partial B_{y}}{\partial z}=\frac{(1-\kappa / 2)}{1-3 \kappa / 2} \frac{\partial E_{x}}{\partial t} .
$$

We make an rearrangement and

$$
\frac{\partial E_{x}}{\partial t}=\frac{1-3 \kappa / 2}{(1-\kappa / 2)}(\nabla \times \mathbf{B})_{x} .
$$

Exploring (49) we have an unusual behavior. The influence of our Lorentz violating background makes a singular response to a nonstationary electrical field. To analyze this behavior we have to mention that a study of the causality and unitarity of this theory was made in [45] and we have established a domain of the value of $\kappa(\kappa \in(2-2 \sqrt{2}, 2))$ for the predictability of this theory. This is the interval in which we assure causality and unitarity. Taking into account (49) we observe that $\lim _{\kappa \rightarrow 2^{-}}((1-3 \kappa / 2) /(1-\kappa / 2))=+\infty$ and we have a singularity. As we are dealing with an effective field theory, this singularity shows that, at this point, we reach the limit of validity of this effective theory that manifests itself in the emergence of this singularity.

6.1. Linearly Polarized Case. Considering the electromagnetic wave propagating in the $z$ direction, we have from (43) and (44)

$$
\begin{aligned}
& \frac{\partial E_{z}}{\partial z}=0 \\
& \frac{\partial B_{z}}{\partial z}=0
\end{aligned}
$$

we can then consider for simplicity $E_{z}=0, B_{z}=0$. Taking the $x$-component of the electric field as monochromatic wave propagation linearly polarized in the $z$ direction

$$
\begin{aligned}
& E_{x}(z, t)=E_{x, 0} \sin (k z-\omega t), \\
& E_{y}(z, t)=E_{y, 0} \sin (k z-\omega t) .
\end{aligned}
$$

We then have from (48)

$$
\begin{aligned}
-\frac{\partial B_{y}(z, t)}{\partial z} & =\frac{(1-\kappa / 2)}{1-3 \kappa / 2} \frac{\partial E_{x}(z, t)}{\partial t} \\
& =-\omega \frac{(1-\kappa / 2)}{1-3 \kappa / 2} E_{x, 0} \cos (k z-\omega t) .
\end{aligned}
$$


The component of the magnetic field will be given by

$$
B_{y}(z, t)=B_{y, 0} \sin (k z-\omega t),
$$

from which the relation between electric and magnetic components will be

$$
B_{y, 0}=\frac{\omega}{k} \frac{(1-\kappa / 2)}{1-3 \kappa / 2} E_{x, 0} .
$$

Notice that there is a deformation factor in the component of the magnetic field due to the presence of Lorentz violation (Figure 1). For the other component

$$
\frac{\partial B_{x}(z, t)}{\partial z}=\frac{\partial E_{y}(z, t)}{\partial t}=-\omega E_{y, 0} \cos (k z-\omega t) .
$$

We then have

$$
B_{x}(z, t)=B_{x, 0} \sin (k z-\omega t),
$$

then resulting in

$$
\begin{aligned}
k B_{x, 0} \cos (k z-\omega t) & =\frac{\partial E_{y}(z, t)}{\partial t} \\
& =-\omega E_{y, 0} \cos (k z-\omega t) .
\end{aligned}
$$

And we have

$$
B_{x, 0}=-\frac{\omega}{k} E_{y, 0}
$$

We then have the magnetic field given in terms of its components:

$$
\begin{aligned}
& B_{x}(z, t)=-\frac{\omega}{k} E_{y, 0} \sin (k z-\omega t), \\
& B_{y}(z, t)=\frac{\omega}{k} \frac{(1-\kappa / 2)}{1-3 \kappa / 2} E_{x, 0} \sin (k z-\omega t) .
\end{aligned}
$$

We can calculate the scalar product between the electric and magnetic field

$$
\begin{aligned}
\mathbf{B} \cdot \mathbf{E}= & B_{x}(z, t) E_{x}(z, t)+B_{y}(z, t) E_{y}(z, t) \\
= & -\frac{\omega}{k} E_{y, 0} E_{x, 0} \sin ^{2}(k z-\omega t) \\
& +\frac{\omega}{k} \frac{(1-\kappa / 2)}{1-3 \kappa / 2} E_{y, 0} E_{x, 0} \sin ^{2}(k z-\omega t)
\end{aligned}
$$

and after simplification

$$
\mathbf{E}(z, t) \cdot \mathbf{B}(z, t)=\frac{\omega}{k} E_{x, 0} E_{y, 0} \frac{\kappa}{1-3 \kappa / 2} \sin ^{2}(k z-\omega t) .
$$

This result shows that the effect of Lorentz violation term $\kappa$ is to make the electric and magnetic field nonorthogonal (Figure 2). Instead of being orthogonal for any time the angles between the electric and magnetic fields are oscillating on time. This is in clear contrast to the classical picture of preserved Lorentz symmetry.

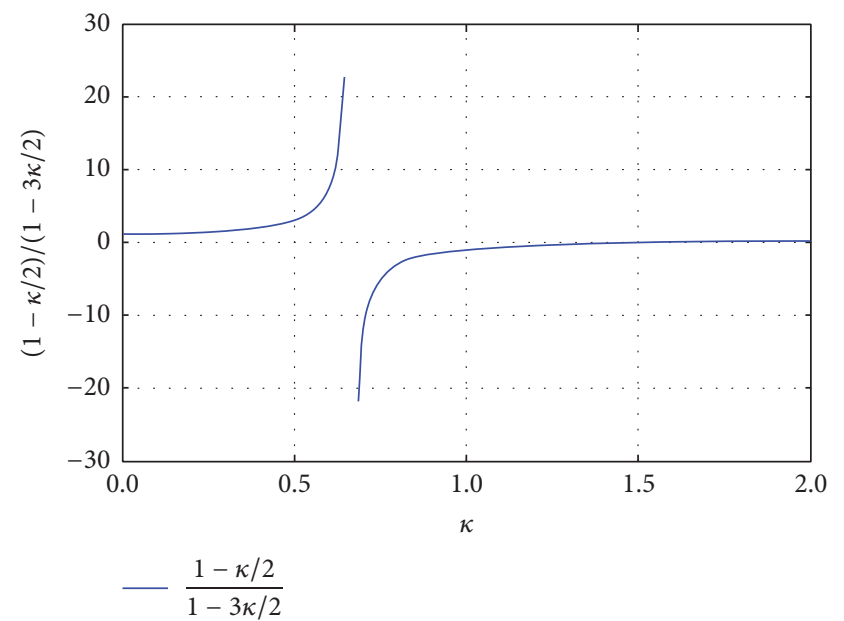

FIGURE 1: (Color online) term of amplitude dependence on $\kappa$.

6.2. Circularly Polarized Case. We can also consider the circularly polarized case, taking the electric field given by

$$
\begin{aligned}
& E_{x}(z, t)=E_{0} \cos (k z-\omega t), \\
& E_{y}(z, t)=E_{0} \sin (k z-\omega t) .
\end{aligned}
$$

As before, the component in the direction of propagation is zero. We can also write

$$
\mathbf{E}(z, t)=E_{0} \widehat{\mathbf{r}}(k z-\omega t),
$$

where

$$
\widehat{\mathbf{r}}(k z-\omega t)=\cos (k z-\omega t) \widehat{\mathbf{x}}+\sin (k z-\omega t) \widehat{\mathbf{y}} .
$$

We then have

$$
\begin{aligned}
-\frac{\partial B_{y}(z, t)}{\partial z} & =\frac{(1-\kappa / 2)}{1-3 \kappa / 2} \frac{\partial E_{x}(z, t)}{\partial t} \\
& =\omega \frac{(1-\kappa / 2)}{1-3 \kappa / 2} E_{0} \sin (k z-\omega t), \\
\frac{\partial B_{x}(z, t)}{\partial z} & =\frac{\partial E_{y}(z, t)}{\partial t}=-\omega E_{0} \cos (k z-\omega t) .
\end{aligned}
$$

The components of the magnetic field will be

$$
\begin{aligned}
& B_{x}(z, t)=B_{0, x} \sin (k z-\omega t), \\
& B_{y}(z, t)=B_{0, y} \cos (k z-\omega t)
\end{aligned}
$$

and then

$$
\begin{aligned}
& B_{0, x}=\frac{\omega}{k} \frac{(1-\kappa / 2)}{1-3 \kappa / 2} E_{0}, \\
& B_{0, y}=-\frac{\omega}{k} E_{0} .
\end{aligned}
$$

We can then write

$$
\begin{aligned}
& B_{x}(z, t)=\frac{\omega}{k} \frac{(1-\kappa / 2)}{1-3 \kappa / 2} E_{0} \sin (k z-\omega t), \\
& B_{y}(z, t)=-\frac{\omega}{k} E_{0} \cos (k z-\omega t) .
\end{aligned}
$$



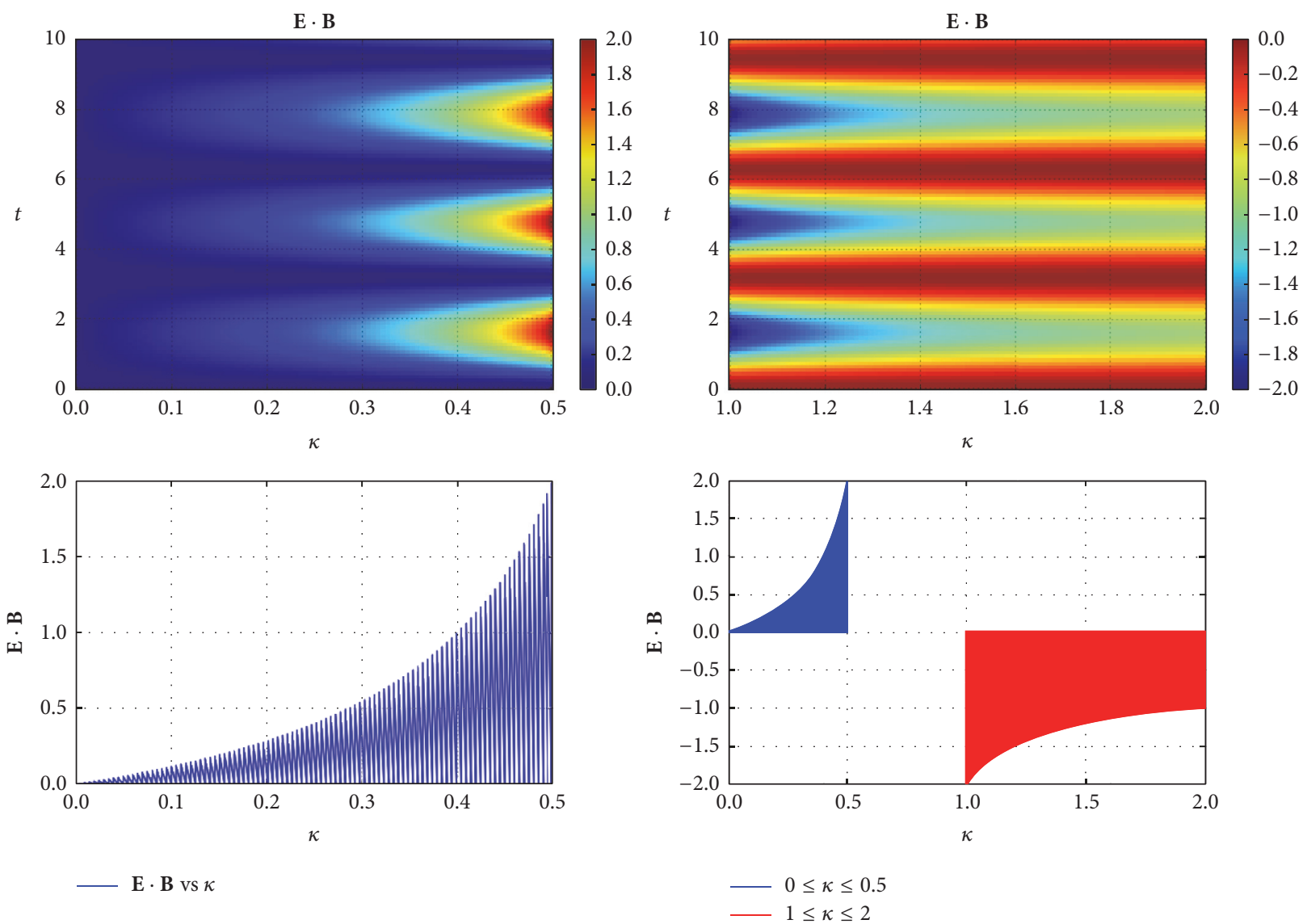

FIGURE 2: (Color online) behavior of the scalar product of the linearly polarized electric field with the magnetic fields with dependence on $\kappa$, considering $0 \leq \kappa \leq 1 / 2$ and $1 \leq \kappa \leq 2$.

Then the electric and magnetic fields are

$$
\begin{aligned}
\mathbf{E}(z, t)= & E_{0} \cos (k z-\omega t) \widehat{\mathbf{x}}+E_{0} \sin (k z-\omega t) \widehat{\mathbf{y}}, \\
\mathbf{B}(z, t)= & \frac{\omega}{k} \frac{(1-\kappa / 2)}{1-3 \kappa / 2} E_{0} \sin (k z-\omega t) \widehat{\mathbf{x}} \\
& -\frac{\omega}{k} E_{0} \cos (k z-\omega t) \widehat{\mathbf{y}} .
\end{aligned}
$$

In this case we have

$$
\begin{aligned}
\mathbf{E}(z, t) & \cdot \mathbf{B}(z, t) \\
= & \frac{\omega}{k} \frac{(1-\kappa / 2)}{1-3 \kappa / 2} E_{0}^{2} \cos (k z-\omega t) \sin (k z-\omega t) \\
& -\frac{\omega}{k} E_{0}^{2} \cos (k z-\omega t) \sin (k z-\omega t)
\end{aligned}
$$

or explicitly

$$
\begin{aligned}
& \mathbf{E}(z, t) \cdot \mathbf{B}(z, t) \\
& \quad=\frac{\kappa}{1-3 \kappa / 2} \frac{\omega}{k} E_{0}^{2} \cos (k z-\omega t) \sin (k z-\omega t) .
\end{aligned}
$$

We note that the dependence on $\kappa$ makes the electric and magnetic fields nonorthogonal (Figure 3) and the elliptical polarization of magnetic field depend on $\kappa$ (Figure 4). As a consequence, the eccentricity of the ellipse in the magnetic field is $\kappa$-dependent as depicted in Figure 5. The restriction of $\kappa$ to the interval $0 \leq \kappa \leq 2$ respects the limits for unitarity found in [45].

\section{Conclusion}

We investigated the influence of CPT-even Lorentz violating contribution of a $K_{F}$-type Lorentz symmetry breaking theory tensor in the Maxwell equations derived from the modified Maxwell action of $K_{F}$-Lorentz violation. We considered the features associated with the derivation of the electromagnetic wave solutions in such a Lorentz violation scenario and considered solutions of the cases of linear and elliptical polarized electromagnetic waves propagating in the vacuum. We showed explicitly that the dependence of $\kappa$ of the nonorthogonality between electric and magnetic fields and that the exhibiting the explicit dependence on $\kappa$.

We showed in this case that the Lorentz violation changes the amplitude of the magnetic field with respect to $\kappa$, without changing the electric field. As a consequence, we showed that the electromagnetic wave fields have a nonorthogonal propagation in the vacuum and exhibited an explicit dependency on the eccentricity of the magnetic field polarization 

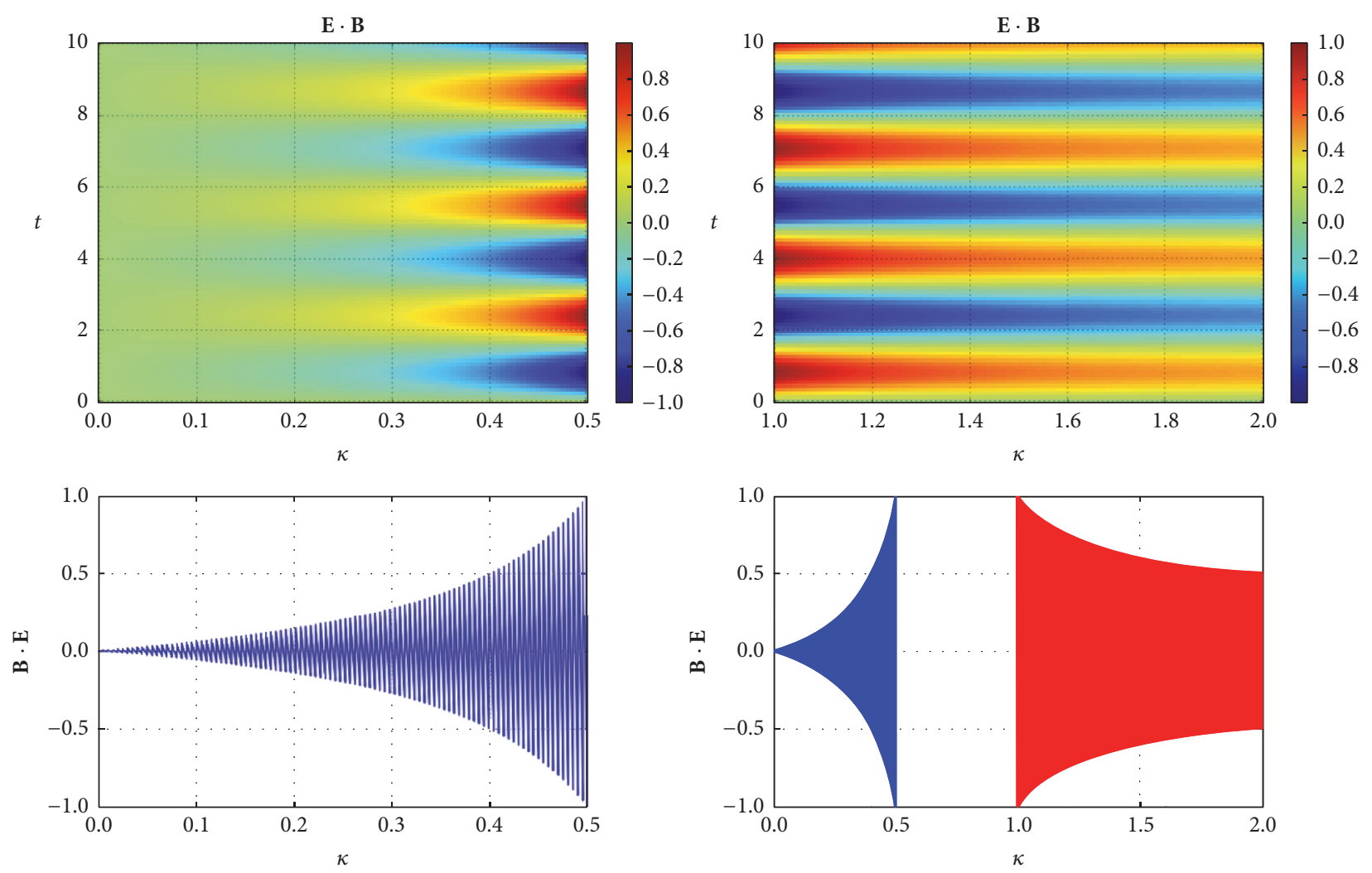

$[\mathbf{B} \cdot \mathbf{E}$ vs $\kappa$

$\begin{aligned} 1 & \leq \kappa \\ - & \leq 2 \\ 0 & \leq 0.5\end{aligned}$

FIGURE 3: (Color online) scalar product of the circularly polarized electric field with the magnetic fields with dependence on $\kappa$, considering $0 \leq \kappa \leq 1 / 2$ and $1 \leq \kappa \leq 2$.

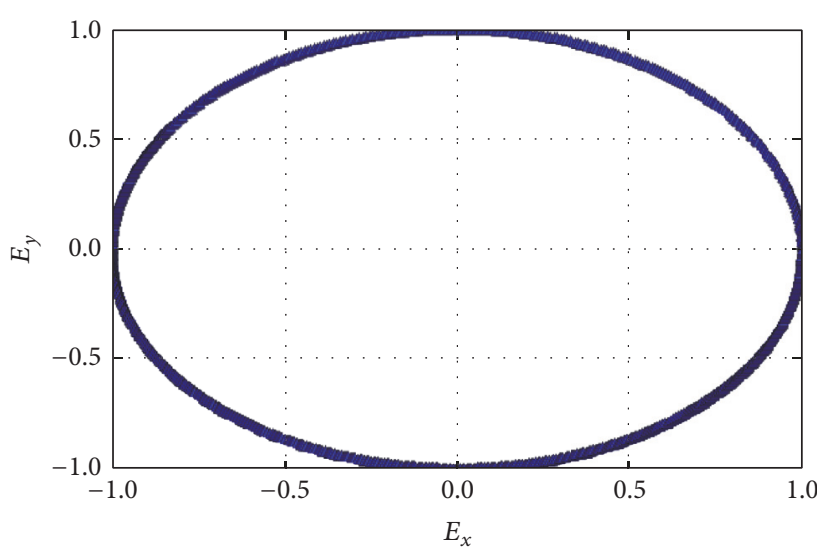

$\bar{\triangle} E_{y}$ vs $E_{x}$

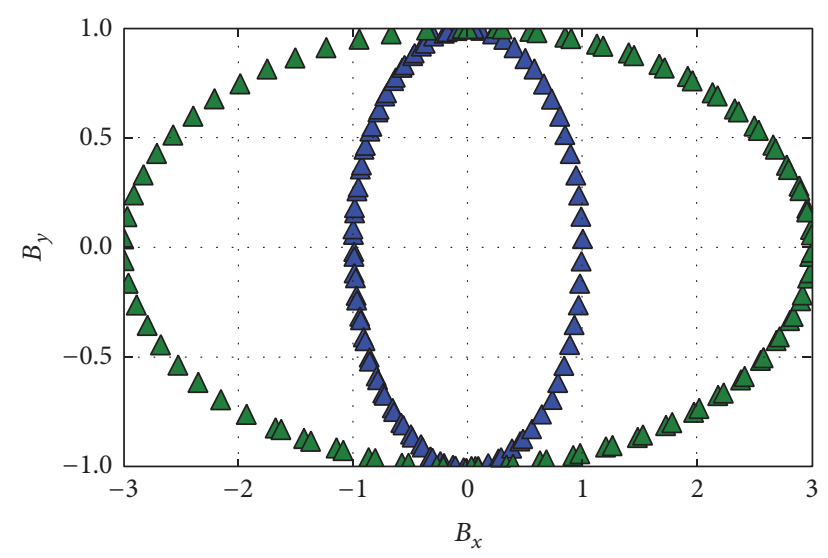

$\Delta \kappa=1 / 2$

$\Delta \kappa=1$

FIGURE 4: (Color online) effect of the $\kappa$ on the elliptical polarization of the magnetic field, for $\kappa=1$ and $\kappa=1 / 2$. 


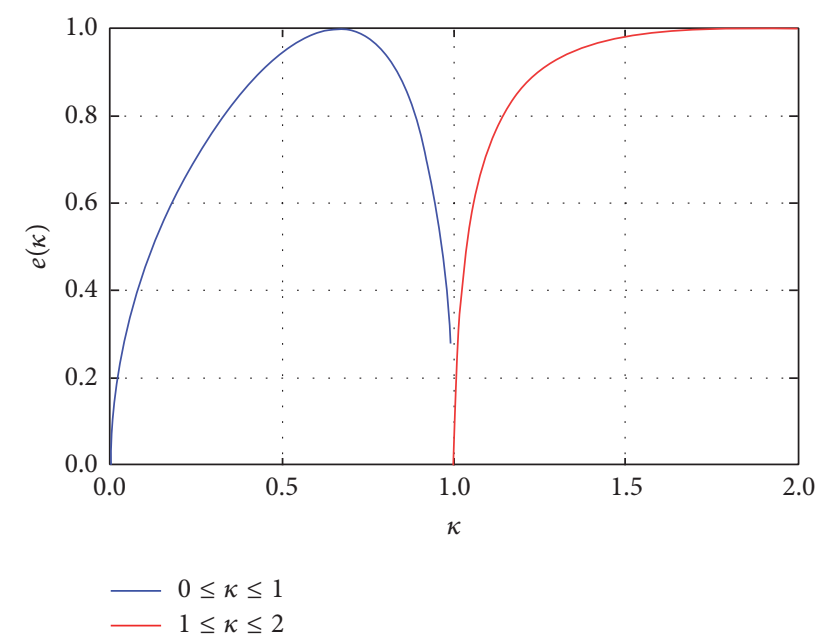

FIgURE 5: (Color online) eccentricity of the ellipse in elliptical polarization of the magnetic field as a function of $\kappa, 0 \leq \kappa \leq 2$.

on $\kappa$-term. Furthermore, we exhibited numerically the consequences of this effect in the cases of linear and elliptical polarization, in particular, the regimes of nonorthogonality of the electromagnetic wave fields and the eccentricity of the elliptical polarization of the magnetic field with dependence on the $\kappa$-term.

\section{Conflicts of Interest}

The authors declare that they have no conflicts of interest.

\section{Acknowledgments}

The authors acknowledge the support by Projects FAPEMAPRONEM-01852/14, FAPEMA-APCINTER-00273/14, Enxoval-UFMA PPPG N03/2014 (Brazil), UFMA-Res. no. 1342-CONSEPE Art1-III-1150/2015-33, UFMA-Res. no. 1342CONSEPE Art1-IV-1151/2015-88, FAPEMA-UNIVERSAL01401/16, and CNPq (Brazil). Thiago Prudêncio also thanks Federal University of Espirito Santo (UFES) for the hospitality in his visit, where this work was concluded as part of the collaboration FAPEMA PRONEM-01852/14.

\section{References}

[1] B. Graner, Y. Chen, E. G. Lindahl, and B. R. Heckel, "Reduced limit on the permanent electric dipole moment of ${ }^{199} \mathrm{Hg}$," Physical Review Letters, vol. 116, no. 16, Article ID 161601, 2016.

[2] J. Baron, W. C. Campbell, D. DeMille et al., "Order of magnitude smaller limit on the electric dipole moment of the electron," Science, vol. 343, no. 6168, pp. 269-272, 2014.

[3] W. Bernreuther and M. Suzuki, "The electric dipole moment of the electron," Reviews of Modern Physics, vol. 63, no. 2, pp. 313340, 1991.

[4] V. A. Kostelecký and S. Samuel, "Phenomenological gravitational constraints on strings and higher-dimensional theories," Physical Review Letters, vol. 63, no. 3, pp. 224-227, 1989.

[5] J. Fröhlich, G. Morchio, and F. Strocchi, "Infrared problem and spontaneous breaking of the Lorentz group in QED," Physics Letters B, vol. 89, no. 1, pp. 61-64, 1979.
[6] A. P. Balachandran and S. Vaidya, "Spontaneous Lorentz violation in gauge theories," The European Physical Journal Plus, vol. 128, article 118, 2013.

[7] A. P. Balachandran, S. Kurkcuoglu, and A. R. de Queiroz, "Spontaneous breaking of Lorentz symmetry and vertex operators for vortices," Modern Physics Letters A, vol. 28, no. 8, Article ID 1350028, 15 pages, 2013.

[8] A. P. Balachandran, S. Kürkçüoglu, A. R. de Queiroz, and S. Vaidya, "Spontaneous Lorentz violation: the case of infrared QED," The European Physical Journal C, vol. 75, no. 2, article 89, 2015.

[9] A. Jenkins, "Spontaneous breaking of Lorentz invariance," Physical Review D, vol. 69, no. 10, Article ID 105007, 2004.

[10] V. A. Kostelecký and S. Samuel, "Spontaneous breaking of Lorentz symmetry in string theory," Physical Review D, vol. 39, no. 2, pp. 683-685, 1989.

[11] Q. G. Bailey, V. A. Kostelecký, and R. Xu, "Short-range gravity and Lorentz violation," Physical Review D, vol. 91, no. 2, Article ID 022006, 6 pages, 2015.

[12] S. M. Carroll and E. A. Lim, "Lorentz-violating vector fields slow the universe down," Physical Review D, vol. 70, no. 12, Article ID 123525, 2004.

[13] D. Colladay and V. A. Kostelecký, "Lorentz-violating extension of the standard model," Physical Review D, vol. 58, no. 11, Article ID 116002, 1998.

[14] V. A. Kostelecký and M. Mewes, "Signals for Lorentz violation in electrodynamics," Physical Review D, vol. 66, no. 5, Article ID 056005, 2002.

[15] M. Schreck, "Quantum field theory based on birefringent modified Maxwell theory," Physical Review D, vol. 89, no. 8, Article ID 085013, 2014.

[16] M. Cambiaso, R. Lehnert, and R. Potting, "Asymptotic states and renormalization in Lorentz-violating quantum field theory," Physical Review D, vol. 90, no. 6, Article ID 065003, 2014.

[17] M. Gomes, J. R. Nascimento, E. Passos, A. Y. Petrov, and A. J. da Silva, "Induction of the four-dimensional Lorentz-breaking non-abelian Chern-Simons action," Physical Review D, vol. 76, no. 4, Article ID 047701, 2007.

[18] M. Gomes, J. R. Nascimento, A. Yu. Petrov, and A. J. da Silva, "Aetherlike Lorentz-breaking actions," Physical Review D, vol. 81, no. 4, Article ID 045018, 2010.

[19] R. Casana, M. M. Ferreira, E. Passos, F. E. P. dos Santos, and E. O. Silva, "New $C P T$-even and Lorentz-violating nonminimal coupling in the Dirac equation," Physical Review D, vol. 87, no. 4, Article ID 047701, 2013.

[20] R. Casana and G. Lazar, "Topological charged BPS vortices in Lorentz-violating Maxwell-Higgs electrodynamics," Physical Review D, vol. 90, no. 6, Article ID 065007, 2014.

[21] R. Casana, M. M. Ferreira Jr., and F. E. P. dos Santos, "GuptaBleuler quantization of the anisotropic parity-even and CPTeven electrodynamics of a standard model extension," Physical Review D, vol. 90, no. 10, Article ID 105025, 6 pages, 2014.

[22] R. Casana, C. F. Farias, and M. M. Ferreira Jr., "Topological selfdual configurations in a Lorentz-violating gauged $O(3)$ sigma model," Physical Review D, vol. 92, Article ID 125024, 2015.

[23] J. B. Araujo, R. Casana, and M. M. Ferreira Jr., "Constraining CPT-even and Lorentz-violating nonminimal couplings with the electron magnetic and electric dipole moments," Physical Review D, vol. 92, no. 2, Article ID 025049, 2015. 
[24] J. R. Nascimento, E. Passos, A. Yu. Petrov, and F. A. Brito, "Lorentz-CPT violation, radiative corrections and finite temperature," Journal of High Energy Physics, vol. 2007, no. 6, article 016, 2007.

[25] A. P. Baeta Scarpelli, T. Mariz, J. R. Nascimento, and A. Y. Petrov, "Four-dimensional aether-like Lorentz-breaking QED revisited and problem of ambiguities," European Physical Journal C, vol. 73, no. 8, article 2526, 2013.

[26] R. Casana, M. M. Ferreira, R. V. Maluf, and F. E. P. dos Santos, "Radiative generation of the CPT-even gauge term of the SME from a dimension-five nonminimal coupling term," Physics Letters B, vol. 726, no. 4-5, pp. 815-819, 2013.

[27] R. Casana, M. M. Ferreira Jr., V. E. Mouchrek-Santos, and E. O. Silva, "Generation of geometrical phases and persistent spin currents in 1-dimensional rings by lorentz-violating terms," Physics Letters B, vol. 746, pp. 171-177, 2015.

[28] K. Bakke and C. Furtado, "Geometric phase for a neutral particle in rotating frames in a cosmic string spacetime," Physical Review D, vol. 80, Article ID 024033, 2009.

[29] K. Bakke and C. Furtado, "Holonomic quantum computation based on the scalar Aharonov-Bohm effect for neutral particles and linear topological defects," Annals of Physics, vol. 327, no. 2, pp. 376-385, 2012.

[30] H. F. Mota and K. Bakke, "Noninertial effects on the ground state energy of a massive scalar field in the cosmic string spacetime," Physical Review D, vol. 89, no. 2, Article ID 027702, 2014.

[31] K. Bakke and C. Furtado, "Quantum holonomies for an electric dipole moment," Physics Letters A, vol. 375, no. 45, pp. 39563959, 2011.

[32] K. Bakke and C. Furtado, "Holonomic Quantum Computation with the Aharonov-Casher setup associated with topological defects," Quantum Information and Computation, vol. 11, p. 444, 2011.

[33] K. Bakke, A. Y. Petrov, and C. Furtado, "A Kaluza-KLEin description of geometric phases in graphene," Annals of Physics, vol. 327, no. 12, pp. 2946-2954, 2012.

[34] K. Bakke and C. Furtado, "One-qubit quantum gates associated with topological defects in solids," Quantum Information Processing, vol. 12, no. 1, pp. 119-128, 2013.

[35] A. G. de Lima, H. Belich, and K. Bakke, "Scalar AharonovBohm-type effect induced by Lorentz symmetry breaking effects," Annalen der Physik, vol. 526, no. 11-12, pp. 514-519, 2014.

[36] K. Bakke and H. Belich, "On the Lorentz symmetry breaking effects on a Dirac neutral particle inside a two-dimensional quantum ring," The European Physical Journal Plus, vol. 129, no. 7, article 147, 2014.

[37] H. Belich and K. Bakke, "A spin-orbit coupling for a neutral particle from Lorentz symmetry breaking effects in the CPT-odd sector of the Standard Model Extension," International Journal of Modern Physics A, vol. 30, no. 22, Article ID 1550136, 2015.

[38] K. Bakke and H. Belich, "On a relativistic scalar particle subject to a Coulomb-type potential given by Lorentz symmetry breaking effects," Annals of Physics, vol. 360, pp. 596-604, 2015.

[39] H. Belich and K. Bakke, "Geometric quantum phases from Lorentz symmetry breaking effects in the cosmic string spacetime," Physical Review D, vol. 90, no. 2, Article ID 025026, 2014.

[40] H. Belich and K. Bakke, "A spin-orbit coupling for a neutral particle from Lorentz symmetry breaking effects in the CPTodd sector of the standard model extension," International Journal of Modern Physics A, vol. 30, no. 22, Article ID 1550136, 2015.
[41] K. Bakke and H. Belich, "A Landau-type quantization from a Lorentz symmetry violation background with crossed electric and magnetic fields," Journal of Physics G: Nuclear and Particle Physics, vol. 42, no. 9, Article ID 095001, 2015.

[42] R. Bluhm, "Breaking Lorentz symmetry," Physics World, vol. 17, no. 3, pp. 41-46, 2004.

[43] M. Pospelov and M. Romalis, "Lorentz invariance on trial," Physics Today, vol. 57, p. 40, 2004.

[44] H. Belich, T. Costa-Soares, M. Santos, and M. Orlando, "Violação da simetria de Lorentz," Revista Brasileira de Ensino de Física, vol. 29, no. 1, pp. 57-64, 2007.

[45] H. Belich, F. J. L. Leal, H. L. C. Louzada, and M. T. D. Orlando, "Investigation of the $K_{F}$-type Lorentz-symmetry breaking gauge models with vortexlike configurations," Physical Review D, vol. 86, no. 12, Article ID 125037, 2012.

[46] O. W. Greenberg, " $C P T$ violation implies violation of Lorentz invariance," Physical Review Letters, vol. 89, no. 23, Article ID 231602, 4 pages, 2002.

[47] E. Kant, F. R. Klinkhamer, and M. Schreck, "Lorentz violation and black-hole thermodynamics: compton scattering process," Physics Letters B, vol. 682, no. 3, pp. 316-321, 2009.

[48] G. Betschart, E. Kant, and F. R. Klinkhamer, "Lorentz violation and black-hole thermodynamics," Nuclear Physics B, vol. 815, no. 1-2, pp. 198-214, 2009.

[49] Q. G. Bailey and V. A. Kostelecký, "Lorentz-violating electrostatics and magnetostatics," Physical Review D, vol. 70, no. 7, Article ID 076006, 2004. 

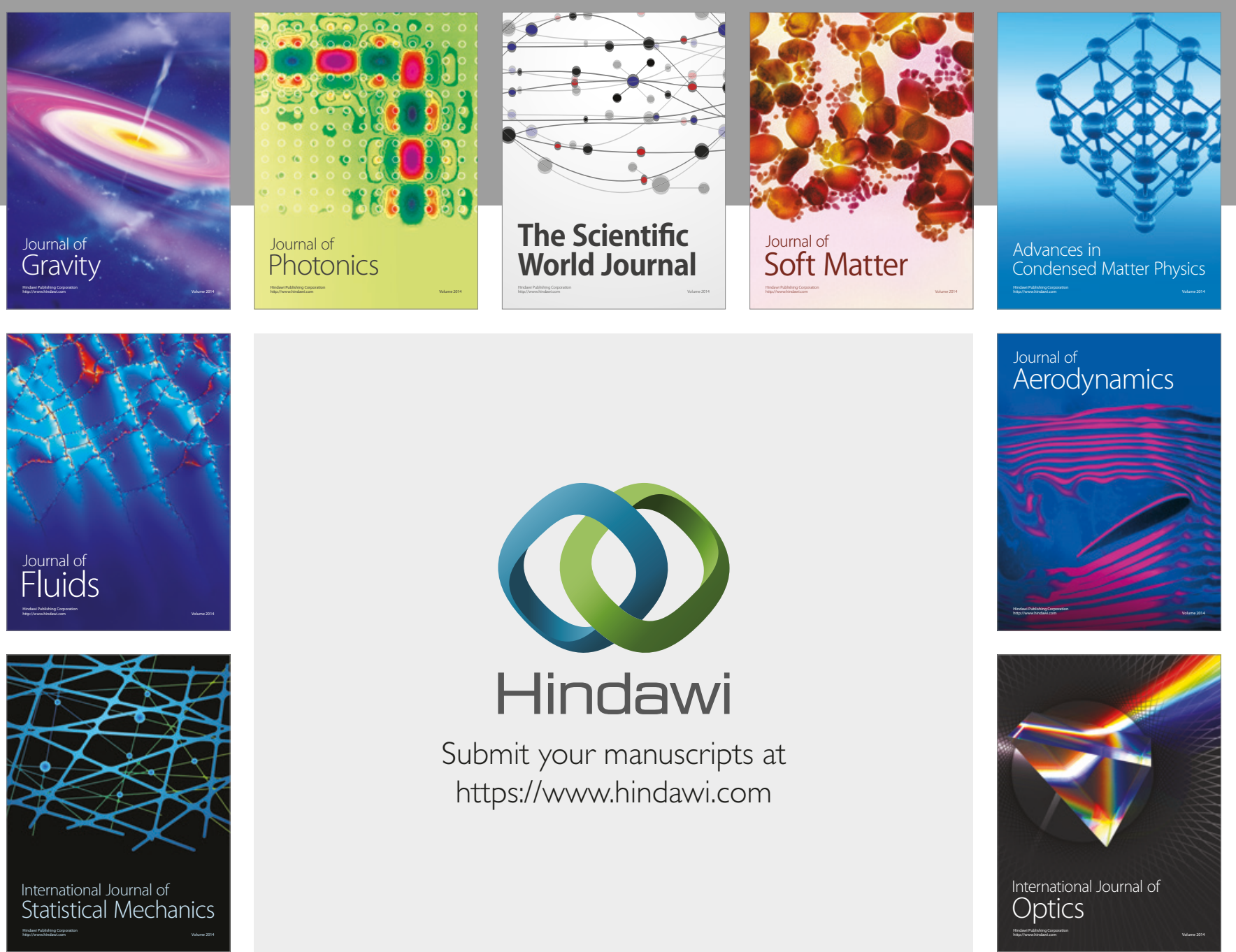

Submit your manuscripts at

https://www.hindawi.com
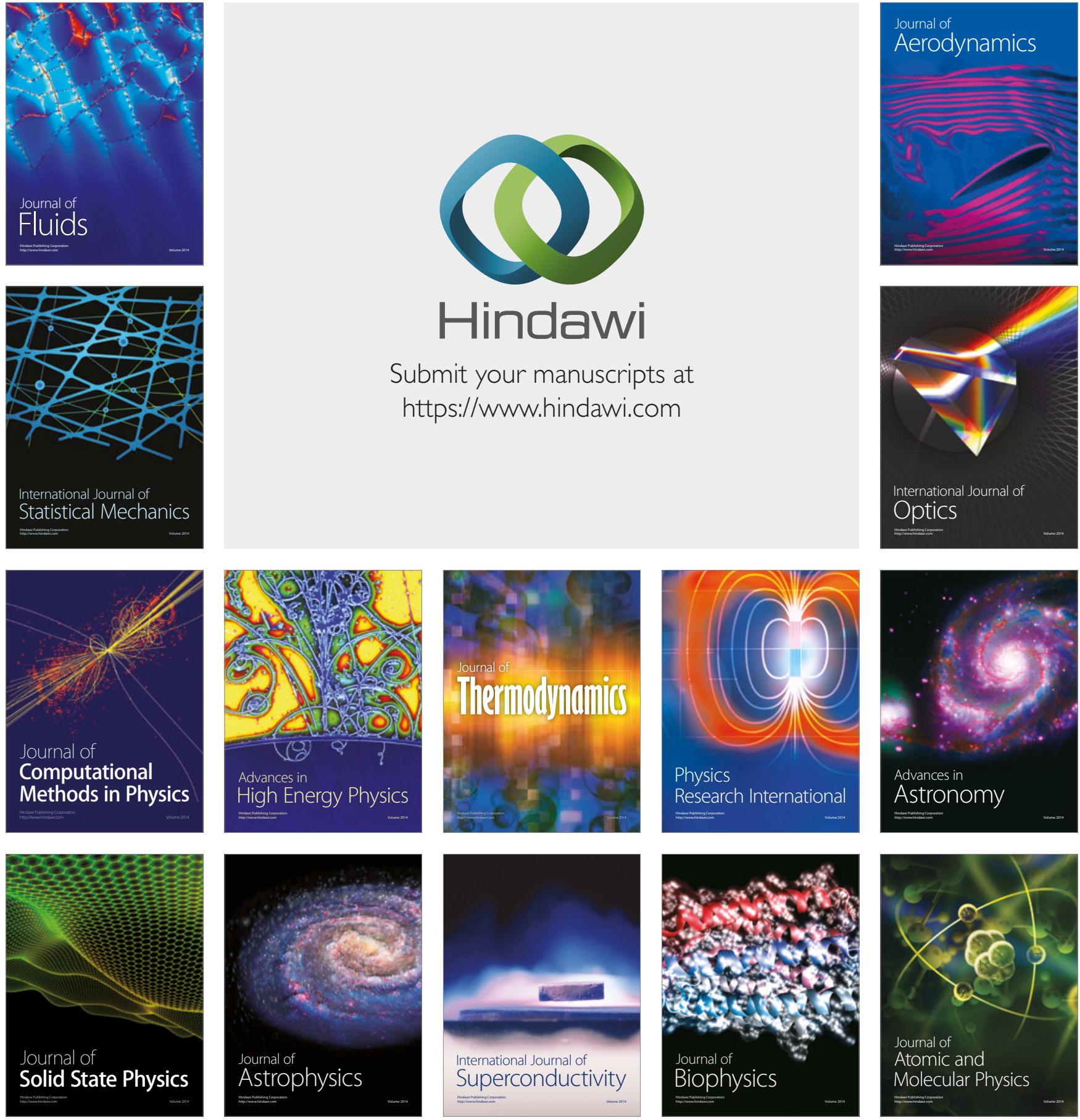